Portland State University

PDXScholar

4-7-2015

\title{
Stochastic Comparisons of Weighted Sums of Arrangement Increasing Random Variables
}

\author{
Xiaoqing Pan \\ University of Science and Technology of China \\ Min Yuan \\ University of Science and Technology of China \\ Subhash C. Kochar \\ Portland State University, kochar@pdx.edu
}

Follow this and additional works at: https://pdxscholar.library.pdx.edu/mth_fac

Part of the Mathematics Commons

Let us know how access to this document benefits you.

\section{Citation Details}

Pan, X., Yuan, M., Kochar, S.C., Stochastic comparisons of weighted sums of arrangement increasing random variables. Statistics and Probability Letters (2015), http://dx.doi.org/10.1016/j.spl.2015.03.012

This Post-Print is brought to you for free and open access. It has been accepted for inclusion in Mathematics and Statistics Faculty Publications and Presentations by an authorized administrator of PDXScholar. Please contact us if we can make this document more accessible: pdxscholar@pdx.edu. 


\title{
Stochastic comparisons of weighted sums of arrangement increasing random variables
}

\author{
Xiaoqing Pan \\ Department of Statistics and Finance, School of Management \\ University of Science and Technology of China \\ Hefei, Anhui 230026 \\ People's Republic of China \\ panxq@ustc.edu.cn \\ Min Yuan \\ Department of Probability and Statistics, School of Mathematics \\ University of Science and Technology of China \\ Hefei, Anhui 230026 \\ People's Republic of China \\ ] myuan@ustc.edu.cn \\ Subhash C. Kochar \\ Fariborz Maseeh Department of Mathematics and Statistics \\ Portland State University, Portland, OR 97006, USA \\ kochar@pdx.edu
}

August, 2014

Revised March, 2015 


\begin{abstract}
Assuming that the joint density of random variables $X_{1}, X_{2}, \ldots, X_{n}$ is arrangement increasing (AI), we obtain some stochastic comparison results on weighted sums of $X_{i}$ 's under some additional conditions. An application to optimal capital allocation is also given.
\end{abstract}

Mathematics Subject Classifications (2000): 60E15; 62N05; $62 \mathrm{G} 30$

Keywords: Increasing convex order; Usual stochastic order; Majorization; Supermodular [Submodular]; Arrangement increasing; Joint likelihood ratio ordering; Log-concavity. 


\section{Introduction}

During the past few decades, linear combinations of random variables have been extensively studied in statistics, operations research, reliability theory, actuarial science and other fields. Most of the related work restricts to some specific distributions such as Exponential, Weibull, Gamma and Uniform, among others. Karlin and Rinott (1983) and Yu (2011) studied the stochastic properties of linear combinations of independent and identically distributed (i.i.d) random variables without putting any distributional assumptions. Later on, $\mathrm{Xu}$ and $\mathrm{Hu}$ (2011, 2012), Pan et al. (2013) and Mao et al. (2013) weakened the i.i.d assumption to independent, yet possibly non-identically distributed (i.ni.d), random variables. It should be noted that most of the related work assumes that the random variables are mutually independent.

Recently, some work has appeared on stochastic comparisons of dependent random variables. $\mathrm{Xu}$ and $\mathrm{Hu}$ (2012) discussed stochastic comparisons of comonotonic random variables with applications to capital allocations. You and Li (2014) focused on linear combinations of random variables with Achimedean dependence structure. Cai and Wei (2014) proposed several new notions of dependence to measure dependence between risks. They proved that characterizations of these notions are related to properties of arrangement increasing (AI) functions (to be defined in Section 2). Motivated by the importance of AI functions, we study the problem of stochastic comparisons of weighted sums of AI random variables in this paper.

We say $X_{1}, \ldots, X_{n}$ are AI random variables if their joint density $f(\boldsymbol{x})$ is an AI function. Ma (2000) proved the following result for AI random variables $X_{1}, \ldots, X_{n}$ :

$$
\mathbf{a} \succeq_{\mathrm{m}} \mathbf{b} \Longrightarrow \sum_{i=1}^{n} a_{(i)} X_{i} \geq_{\mathrm{icx}} \sum_{i=1}^{n} b_{(i)} X_{i}, \quad \forall \mathbf{a}, \mathbf{b} \in \Re^{n},
$$

where $a_{(1)} \leq a_{(2)} \leq \cdots \leq a_{(n)}$ is the increasing arrangement of the components of the vector $\mathbf{a}=\left(a_{1}, a_{2}, \ldots, a_{n}\right)$. The formal definitions of stochastic orders and majorization orders are given in Section 2.

Let $X_{1}, X_{2}, \ldots, X_{n}$ be independent random variables satisfying

$$
X_{1} \geq_{\mathrm{hr}} X_{2} \geq_{\mathrm{hr}} \cdots \geq_{\mathrm{hr}} X_{n}
$$

and let $\phi(x, a)$ be a convex function which is increasing in $x$ for each $a$. Mao et al. (2013) proved that

(i) if $\phi$ is submodular, then

$$
\mathbf{a} \succeq_{\mathrm{m}} \mathbf{b} \Longrightarrow \sum_{i=1}^{n} \phi\left(X_{i}, a_{(i)}\right) \geq_{\mathrm{icx}} \sum_{i=1}^{n} \phi\left(X_{i}, b_{(i)}\right) ;
$$


(ii) if $\phi$ is supermodular, then

$$
\mathbf{a} \succeq_{\mathrm{m}} \mathbf{b} \Longrightarrow \sum_{i=1}^{n} \phi\left(X_{i}, a_{(n-i+1)}\right) \geq_{\mathrm{icx}} \sum_{i=1}^{n} \phi\left(X_{i}, b_{(n-i+1)}\right) .
$$

The function $\phi$ in (1.2) and (1.3) could be interpreted as some appropriate distance measures in actuarial science. For more details, please refer to Xu and $\mathrm{Hu}$ (2012).

In this paper we further study the problem of stochastic comparisons of linear combinations of AI random variables not only for increasing convex ordering, but also for the usual stochastic ordering. The rest of this paper is organized as follows. Some preliminaries are given in Section 2. The main results are presented in Section 3. An application to optimal capital allocation is discussed in Section 4.

\section{Preliminaries}

In this section, we give definitions of some stochastic orders, majorization orders and supermodular [submodular] functions. Throughout the paper, the terms 'increasing' and 'decreasing' are used to mean 'non-decreasing' and 'non-increasing', respectively.

Definition 2.1 (Stochastic orders)

Let $X$ and $Y$ be two random variables with probability (mass) density functions $f$ and $g$; and survival functions $\bar{F}$ and $\bar{G}$ respectively. We say that $X$ is smaller than $Y$

(1) in the usual stochastic order, denoted by $X \leq_{\mathrm{st}} Y$, if $\bar{F}(t) \leq \bar{G}(t)$ for all $t$ or, equivalently, if $\mathrm{E}[h(X)] \leq \mathrm{E}[h(Y)]$ for all increasing functions $h$;

(2) in the hazard rate order, denoted by $X \leq_{\mathrm{hr}} Y$, if $\bar{G}(t) / \bar{F}(t)$ is increasing in $t$ for which the ratio is well defined;

(3) in the likelihood ratio order, denoted by $X \leq_{\operatorname{lr}} Y$, if $g(t) / f(t)$ is increasing in $t$ for which the ratio is well defined;

(4) in the increasing convex order, denoted by $X \leq_{\mathrm{icx}} Y$, if $\mathrm{E}[h(X)] \leq \mathrm{E}[h(Y)]$ for all increasing convex functions $h$ for which the expectations exist.

The relationships among these orders are shown in the following diagram (see Shaked and Shanthikumar, 2007; Müller and Stoyan, 2002):

$$
X \leq_{\mathrm{lr}} Y \Longrightarrow X \leq_{\mathrm{hr}} Y \Longrightarrow X \leq_{\mathrm{st}} Y \Longrightarrow X \leq_{\mathrm{icx}} Y .
$$

Shanthikumar and Yao (1991) considered the problem of extending the above concepts to compare the components of dependent random variables. In this paper we will focus only on extension of likelihood ratio ordering to the case of dependent random variables. Let $(X, Y)$ be a continuous bivariate random vector on $[0, \infty]^{2}$ with joint density (or mass) function $f(x, y)$. 
Definition 2.2 For a bivariate random variable $(X, Y), X$ is said to be smaller than $Y$ according to joint likelihood ordering, denoted by $X \leq_{\ell r: j} Y$, if and only if

$$
E[\Psi(X, Y)] \geq E[\Psi(Y, X)], \quad \Psi \in \mathcal{G}_{\ell r},
$$

where

$$
\mathcal{G}_{\ell r}:\{\Psi: \Psi(x, y) \geq \Psi(y, x), \quad x \leq y\}
$$

It can be seen that

$$
X \leq_{\ell r: j} Y \Leftrightarrow f \in \mathcal{G}_{\ell r},
$$

where $f(\cdot, \cdot)$ denotes the joint density of $(X, Y)$.

As pointed out by Shanthikumar and Yao (1991), joint likelihood ratio ordering between the components of a bivariate random vector may not imply likelihood ratio ordering between their marginal distributions unless the random variables are independent, but it does imply stochastic ordering between them, that is,

$$
X \leq_{\ell r: j} Y \Rightarrow X \leq_{\mathrm{st}} Y .
$$

A bivariate function $\Psi \in \mathcal{G}_{\ell r}$ is called arrangement increasing (AI). Hollander et al. (1977) have studied many interesting properties of such functions, though, apparently, they did not relate it to the notion of likelihood ratio ordering. We can extend this concept to compare more than two random variables in the following way.

Let $\pi=(\pi(1), \ldots, \pi(n))$ be any permutation of $\{1, \ldots, n\}$ and let $\pi(\mathbf{x})=\left(x_{\pi(1)}, \ldots, x_{\pi(n)}\right)$. For any $1 \leq i \neq j \leq n$, we denote $\pi_{i j}=\left(\pi_{i j}(1), \ldots, \pi_{i j}(n)\right)$ with $\pi_{i j}(i)=j, \pi_{i j}(j)=i$ and $\pi_{i j}(k)=k$ for $k \neq i, j$.

\section{Definition 2.3 (AI function)}

A real-valued function $g(\mathbf{x})$ defined on $\Re^{n}$ is said to be an arrangement increasing (AI) function if

$$
\left(x_{i}-x_{j}\right)\left[g(\mathbf{x})-g\left(\boldsymbol{\pi}_{i j}(\mathbf{x})\right)\right] \leq 0,
$$

for any pair $(i, j)$ such that $1 \leq i<j \leq n$.

We say $X_{1}, \ldots, X_{n}$ are AI random variables if their joint density $f(\boldsymbol{x})$ is an AI function.

Definition 2.4 We say that a function $h(x, y)$ is Totally Positive of order $2\left(T P_{2}\right)$ if $h(x, y) \geq 0$ and

$$
\left|\begin{array}{ll}
h\left(x_{1}, y_{1}\right) & h\left(x_{1}, y_{2}\right) \\
h\left(x_{2}, y_{1}\right) & h\left(x_{2}, y_{2}\right)
\end{array}\right| \geq 0
$$

whenever $x_{1}<x_{2}, y_{1}<y_{2}$. 
Hollander et al. (1977) and Marshall et al. (2011) gave many examples of AI random variables. The following vectors of random variables $\mathbf{X}=\left(X_{1}, \ldots X_{n}\right)$ are arrangement increasing.

(1) $X_{1}, \ldots, X_{n}$ are identically independent distributed random variables.

(2) $X_{1}, \ldots, X_{n}$ are exchangeable random variables.

(3) Suppose $X_{1}, \ldots, X_{n}$ are independent random variables with density functions $h\left(\lambda_{i}, x_{i}\right)$, $i=1, \ldots, n$, respectively. Then $f(\boldsymbol{x})=\prod h\left(\lambda_{i}, x_{i}\right)$ is arrangement increasing if and only if $h(\lambda, x)$ is $T P_{2}$ in $\lambda$ and $x$.

Hollander et al. (1977) have shown that the following multivariate distributions are AI: Multinomial, Negative multinomial, Multivariate hypergeometric, Dirichlet, Inverted Dirichlet, Negative multivariate hypergeometric, Dirichlet compound negative multinomal, Multivariate logarithmic series distribution, Multivariate $F$ distribution, Multivariate Pareto distribution, Multivariate normal distribution with common variance and common covariance.

Majorization defines a partial ordering of the diversity of the components of vectors. For extensive and comprehensive details on the theory of majorization order and their applications, please refer to Marshall et al. (2011).

Definition 2.5 (Majorization, Schur-concave [Schur-convex] and log-concave)

For vectors $\mathbf{x}, \mathbf{y} \in \Re^{n}, \mathbf{x}$ is said to be majorized by $\mathbf{y}$, denoted by $\mathbf{x} \preceq_{\mathrm{m}} \mathbf{y}$, if $\sum_{i=1}^{n} x_{(i)}=\sum_{i=1}^{n} y_{(i)}$ and

$$
\sum_{i=1}^{j} x_{(i)} \geq \sum_{i=1}^{j} y_{(i)} \text { for } j=1, \ldots, n-1 .
$$

A real-valued function $\phi$ defined on a set $A \subseteq \Re^{n}$ is said to be Schur-concave [Schur-convex] on $A$ if, for any $\mathbf{x}, \mathbf{y} \in A$,

$$
\mathbf{x} \succeq_{\mathrm{m}} \mathbf{y} \Longrightarrow \phi(\mathbf{x}) \leq[\geq] \phi(\mathbf{y})
$$

and $\phi$ is said to be log-concave on $A \subset \Re^{n}$ if $A$ is a convex set and, for any $\mathbf{x}, \mathbf{y} \in A$ and $\alpha \in[0,1]$,

$$
\phi(\alpha \mathbf{x}+(1-\alpha) \mathbf{y}) \geq[\phi(\mathbf{x})]^{\alpha}[\phi(\mathbf{y})]^{1-\alpha}
$$

Definition 2.6 (Supermodular [Submodular] function) A real-valued function $\varphi: \Re^{n} \rightarrow \Re$ is said to be supermodular [submodular] if

$$
\varphi(\mathbf{x} \vee \mathbf{y})+\varphi(\mathbf{x} \wedge \mathbf{y}) \geq[\leq] \varphi(\mathbf{x})+\varphi(\mathbf{y}) \text { for all } \mathbf{x}, \mathbf{y} \in \Re^{n}
$$

Here, $\vee$ and $\wedge$ denote the componentwise maximum and the componentwise minimum, respectively. If $\varphi: \Re^{n} \rightarrow \Re$ has second partial derivatives, then it is supermodular [submodular] if and 
only if

$$
\frac{\partial^{2}}{\partial x_{i} \partial x_{j}} \varphi(\mathbf{x}) \geq[\leq] 0 \text { for all } i \neq j \text { and } \mathbf{x} \in \Re^{n} .
$$

Marshall et al. (2011) gave several examples of supermodelar [submodular] functions. Below we give some examples of linear functions which are supermodular [submodular].

(1) $\varphi(\mathbf{a}, \mathbf{x})=\sum_{i=1}^{n} \phi_{1}\left(a_{i}\right) \phi_{2}\left(x_{i}\right)$ : if $\phi_{1}$ and $\phi_{2}$ are increasing, then $\varphi(\mathbf{a}, \mathbf{x})$ is submodular; and if if $\phi_{1}$ is decreasing and $\phi_{2}$ is increasing, then $\varphi(\mathbf{a}, \mathbf{x})$ is supermodular.

(2) $\varphi(\mathbf{a}, \mathbf{x})=\sum_{i=1}^{n} \phi\left(x_{i}-a_{i}\right)$ : if $\phi$ is convex, then $\varphi(\mathbf{a}, \mathbf{x})$ is submodular; and if $\phi$ is concave, then $\varphi(\mathbf{a}, \mathbf{x})$ is supermodular.

(3) $\varphi(\mathbf{a}, \mathbf{x})=\sum_{i=1}^{n} \max \left\{a_{i}, x_{i}\right\}$ is submodular.

(4) $\varphi(\mathbf{a}, \mathbf{x})=\sum_{i=1}^{n} \sup \left\{c_{1} a_{i}+c_{2} x_{i}:\left(c_{1}, c_{2}\right) \in C\right\}$ is submodular, where $C \subseteq \Re^{2}$.

\section{Main results}

In this section, we study stochastic comparisons of weighted sums of the form $\sum_{i=1}^{n} \phi\left(X_{i}, a_{i}\right)$ where $X_{1}, \ldots, X_{n}$ are random variables with joint density function $f(\mathbf{x})$. In what follows, we make the following assumptions:

(A1) $f(\mathbf{x})$ is log-concave,

(A2) $f(\mathbf{x})$ is arrangement increasing,

(A3) $\phi: \Re^{2} \rightarrow \Re$ is a convex function.

We consider both usual stochastic order as well as increasing convex order for comparison purposes.

\subsection{Usual stochastic ordering}

Before we give the main result, we list several lemmas, which will be used in the sequel.

Lemma 3.1 (Prékopa, 1973; Eaton, 1982)

Suppose that $h: \Re^{m} \times \Re^{k} \rightarrow \Re_{+}$is a log-concave function and that

$$
g(\mathbf{x})=\int_{\Re^{k}} h(\mathbf{x}, \mathbf{z}) d \mathbf{z}
$$

is finite for each $\mathbf{x} \in \Re^{m}$. Then $g$ is log-concave on $\Re^{m}$. 
Lemma 3.2 (Pan et al., 2013)

If $g: \Re^{2} \rightarrow \Re_{+}$is log-concave and $-g$ is AI, i.e.

$$
g\left(x_{(2)}, x_{(1)}\right) \geq g\left(x_{(1)}, x_{(2)}\right) \quad \text { for all }\left(x_{1}, x_{2}\right) \in \Re^{2},
$$

then

$$
\left(x_{1}, x_{2}\right) \preceq_{\mathrm{m}}\left(y_{1}, y_{2}\right) \Longrightarrow g\left(x_{(1)}, x_{(2)}\right) \geq g\left(y_{(1)}, y_{(2)}\right) .
$$

We list Theorem 23 in Karlin and Rinott (1983) as a lemma, and we give a new proof as follows.

Lemma 3.3 (Karlin and Rinott, 1983)

Let $\mathbf{X} \in \Re^{n}$ have a log-concave density and let $\phi(\mathbf{x}, \mathbf{a})$ be convex in $(\boldsymbol{x}, \boldsymbol{a}) \in \Re^{n+m}$. Then $g(\mathbf{a})=P(\phi(\mathbf{X}, \mathbf{a}) \leq t)$ is log-concave on $\Re^{m}$.

Proof. If we denote

$$
A=\left\{(\mathbf{x}, \mathbf{a}) \in \Re^{n+m}: \phi(\mathbf{x}, \mathbf{a}) \leq t\right\},
$$

then $A$ is a convex set due to the convexity of $\phi$. Thus, $\mathrm{I}_{A}$ is log-concave. If $f(\mathbf{x})$ denotes the joint density function of $\mathbf{X}$, then we have

$$
g(\mathbf{a})=\int_{\Re^{n}} f(\mathbf{x}) \mathrm{I}_{A} d \mathbf{x} .
$$

Since $f(\mathbf{x})$ is $\log$-concave, $f(\mathbf{x}) \mathrm{I}_{\mathbf{A}}$ is log-concave. By Lemma $3.1, g(\mathbf{a})$ is log-concave.

From Lemma 3.3, we have

Lemma 3.4 Let $\mathbf{X} \in \Re^{n}$ have a log-concave density and let $\phi(\mathbf{x}, \mathbf{a})$ be concave in $(\boldsymbol{x}, \boldsymbol{a}) \in \Re^{n+m}$. Then $g(\mathbf{a})=P(\phi(\mathbf{X}, \mathbf{a}) \geq t)$ is log-concave on $\Re^{m}$.

Theorem 3.5 Under the assumptions (A1), (A2) and (A3),

(i) if $\phi$ is supermodular, then, for any $\mathbf{a}, \mathbf{b} \in \Re^{n}$,

$$
\mathbf{a} \succeq_{\mathrm{m}} \mathbf{b} \Longrightarrow \sum_{i=1}^{n} \phi\left(X_{i}, a_{(i)}\right) \geq_{\mathrm{st}} \sum_{i=1}^{n} \phi\left(X_{i}, b_{(i)}\right) .
$$

(ii) if $\phi$ is submodular, then, for any $\mathbf{a}, \mathbf{b} \in \Re^{n}$,

$$
\mathbf{a} \succeq_{\mathrm{m}} \mathbf{b} \Longrightarrow \sum_{i=1}^{n} \phi\left(X_{n-i+1}, a_{(i)}\right) \geq_{\text {st }} \sum_{i=1}^{n} \phi\left(X_{n-i+1}, b_{(i)}\right) .
$$

Proof. Since the proof of part (ii) is quite similar to part (i), we only prove part (i). By the nature of majorization, we only need to prove that

$$
\phi\left(X_{1}, a_{(1)}\right)+\phi\left(X_{2}, a_{(2)}\right)+\sum_{i=3}^{n} \phi\left(X_{i}, c_{i}\right) \geq_{\text {st }} \phi\left(X_{1}, b_{(1)}\right)+\phi\left(X_{2}, b_{(2)}\right)+\sum_{i=3}^{n} \phi\left(X_{i}, c_{i}\right)
$$


holds, for all

$$
\left(a_{(1)}, a_{(2)}, c_{3}, \ldots, c_{n}\right) \succeq_{\mathrm{m}}\left(b_{(1)}, b_{(2)}, c_{3}, \ldots, c_{n}\right),
$$

with $\left(a_{(1)}, a_{(2)}\right) \succeq_{\mathrm{m}}\left(b_{(1)}, b_{(2)}\right)$. For any fixed $t$, we denote

$$
g\left(a_{1}, a_{2}\right)=P(\varphi(\mathbf{X}, \mathbf{a}) \leq t)
$$

with

$$
\varphi(\mathbf{x}, \mathbf{a})=\phi\left(x_{1}, a_{1}\right)+\phi\left(x_{2}, a_{2}\right)+\sum_{i=3}^{n} \phi\left(x_{i}, c_{i}\right)
$$

Since $\phi$ is convex, $\varphi(\mathbf{x}, \mathbf{a})$ is convex on $\Re^{2 n}$. By Lemma 3.3, $g\left(a_{1}, a_{2}\right)$ is log-concave. From Lemma 3.2 , it is sufficient to prove (3.1). Hence, we only need to prove

$$
\varphi\left(\mathbf{X}, \mathbf{a}_{21}\right) \leq_{\text {st }} \varphi\left(\mathbf{X}, \mathbf{a}_{12}\right)
$$

where $\mathbf{a}_{12}=\left(a_{(1)}, a_{(2)}\right)$ and $\mathbf{a}_{21}=\left(a_{(2)}, a_{(1)}\right)$. It is sufficient to prove that, for all increasing function $h$,

$$
\mathrm{E}\left[h\left(\varphi\left(\mathbf{X}, \mathbf{a}_{12}\right)\right)\right] \geq \mathrm{E}\left[h\left(\varphi\left(\mathbf{X}, \mathbf{a}_{21}\right)\right)\right]
$$

Now,

$$
\begin{aligned}
\mathrm{E} & {\left[h\left(\varphi\left(\mathbf{X}, \mathbf{a}_{12}\right)\right)\right]-\mathrm{E}\left[h\left(\varphi\left(\mathbf{X}, \mathbf{a}_{21}\right)\right)\right] } \\
= & \left(\int \cdots \int_{x_{1} \leq x_{2}}+\int \cdots \int_{x_{1} \geq x_{2}}\right) h\left(\varphi\left(\mathbf{x}, \mathbf{a}_{12}\right)\right) f(\mathbf{x}) d x_{1} \ldots d x_{n} \\
& -\left(\int \cdots \int_{x_{1} \leq x_{2}}+\int \cdots \int_{x_{1} \geq x_{2}}\right) h\left(\varphi\left(\mathbf{x}, \mathbf{a}_{21}\right)\right) f(\mathbf{x}) d x_{1} \ldots d x_{n} \\
& =\int \cdots \int_{x_{1} \leq x_{2}}\left[h\left(\varphi\left(\mathbf{x}, \mathbf{a}_{12}\right)\right)-h\left(\varphi\left(\mathbf{x}, \mathbf{a}_{21}\right)\right)\right]\left[f(\mathbf{x})-f\left(\boldsymbol{\pi}_{12}(\mathbf{x})\right)\right] d x_{1} \ldots d x_{n} .
\end{aligned}
$$

Since $f(\mathbf{x})$ is AI, it follows that

$$
f(\mathbf{x})-f\left(\boldsymbol{\pi}_{12}(\mathbf{x})\right) \geq 0, \quad \forall x_{1} \leq x_{2} .
$$

Meanwhile, since $\phi$ is supermodular, for all $x_{1} \leq x_{2}$ and $a_{(1)} \leq a_{(2)}$, we have

$$
\phi\left(x_{1}, a_{(1)}\right)+\phi\left(x_{2}, a_{(2)}\right) \geq \phi\left(x_{1}, a_{(2)}\right)+\phi\left(x_{2}, a_{(1)}\right) .
$$

Thus,

$$
\phi\left(x_{1}, a_{(1)}\right)+\phi\left(x_{2}, a_{(2)}\right)+\sum_{i=3}^{n} \phi\left(x_{i}, c_{i}\right) \geq \phi\left(x_{1}, a_{(2)}\right)+\phi\left(x_{2}, a_{(1)}\right)+\sum_{i=3}^{n} \phi\left(x_{i}, c_{i}\right) .
$$

Since $h$ is increasing, we have

$$
h\left(\varphi\left(\mathbf{x}, \mathbf{a}_{12}\right)\right)-h\left(\varphi\left(\mathbf{x}, \mathbf{a}_{21}\right)\right) \geq 0 .
$$


Therefore, (3.2) holds and the desired result follows.

Since exchangeable random variables are arrangement increasing, the following results follows immediately from this theorem.

Corollary 3.6 Let $X_{1}, \ldots, X_{n}$ be exchangeable random variables satisfying assumption (A1). If $\phi$ is a convex function on $\Re$, then, for any $\mathbf{a}, \mathbf{b} \in \Re^{n}$,

$$
\mathbf{a} \succeq_{\mathrm{m}} \mathbf{b} \Longrightarrow \sum_{i=1}^{n} \phi\left(X_{i}, a_{i}\right) \geq_{\mathrm{st}} \sum_{i=1}^{n} \phi\left(X_{i}, b_{i}\right)
$$

Proposition 3.7 Under the assumptions (A1), (A2) and (A3),

(i) if $\phi$ is supermodular, then, for any $\mathbf{a}, \mathbf{b} \in \Re^{n}$,

$$
\mathbf{a} \succeq_{\mathrm{m}} \mathbf{b} \Longrightarrow \sum_{i=1}^{n} \phi\left(X_{i}, a_{(i)}\right) \geq_{\mathrm{st}} \sum_{i=1}^{n} \phi\left(X_{i}, b_{i}\right) .
$$

(ii) if $\phi$ is submodular, then, for any $\mathbf{a}, \mathbf{b} \in \Re^{n}$,

$$
\mathbf{a} \succeq_{\mathrm{m}} \mathbf{b} \Longrightarrow \sum_{i=1}^{n} \phi\left(X_{n-i+1}, a_{(i)}\right) \geq_{\mathrm{st}} \sum_{i=1}^{n} \phi\left(X_{n-i+1}, b_{i}\right)
$$

Proof. The proof of (ii) is quite similar to that of (i), so we only prove (i). By the nature of majorization, we only need to prove that for all

$$
\left(a_{(1)}, a_{(2)}, c_{3}, \ldots, c_{n}\right) \succeq_{\mathrm{m}}\left(b_{(1)}, b_{(2)}, c_{3}, \ldots, c_{n}\right),
$$

with $\left(a_{(1)}, a_{(2)}\right) \succeq_{\mathrm{m}}\left(b_{(1)}, b_{(2)}\right)$, we have

$$
\phi\left(X_{1}, a_{(1)}\right)+\phi\left(X_{2}, a_{(2)}\right)+\sum_{i=3}^{n} \phi\left(X_{i}, c_{i}\right) \geq_{\text {st }} \phi\left(X_{1}, b_{1}\right)+\phi\left(X_{2}, b_{2}\right)+\sum_{i=3}^{n} \phi\left(X_{i}, c_{i}\right) .
$$

From (3.2), we have

$$
\phi\left(X_{1}, b_{(1)}\right)+\phi\left(X_{2}, b_{(2)}\right)+\sum_{i=3}^{n} \phi\left(X_{i}, c_{i}\right) \geq_{\mathrm{st}} \phi\left(X_{1}, b_{(2)}\right)+\phi\left(X_{2}, b_{(1)}\right)+\sum_{i=3}^{n} \phi\left(X_{i}, c_{i}\right) .
$$

Meanwhile, from Theorem 3.5, we have

$$
\phi\left(X_{1}, a_{(1)}\right)+\phi\left(X_{2}, a_{(2)}\right)+\sum_{i=3}^{n} \phi\left(X_{i}, c_{i}\right) \geq_{\text {st }} \phi\left(X_{1}, b_{(1)}\right)+\phi\left(X_{2}, b_{(2)}\right)+\sum_{i=3}^{n} \phi\left(X_{i}, c_{i}\right) .
$$

Combing (3.3) and (3.4), we have

$$
\phi\left(X_{1}, a_{(1)}\right)+\phi\left(X_{2}, a_{(2)}\right)+\sum_{i=3}^{n} \phi\left(X_{i}, c_{i}\right) \geq_{\mathrm{st}} \phi\left(X_{1}, b_{(2)}\right)+\phi\left(X_{2}, b_{(1)}\right)+\sum_{i=3}^{n} \phi\left(X_{i}, c_{i}\right) .
$$

From (3.4) and (3.5), we get the desired result. 
Corollary 3.8 Under the assumptions (A1), (A2) and (A3), for any $\mathbf{a}, \mathbf{b} \in \Re^{n}$,

$$
\mathbf{a} \succeq_{\mathrm{m}} \mathbf{b} \Longrightarrow \sum_{i=1}^{n} \phi\left(X_{n-i+1}-a_{(i)}\right) \geq_{\text {st }} \sum_{i=1}^{n} \phi\left(X_{n-i+1}-b_{i}\right) \text {. }
$$

Proof. It is easy to prove that $\phi(x-a)$ is convex and submodular on $\Re^{2}$. By Proposition 3.7, the result follows.

If the function $\phi$ is concave, we get a similar result in the same way..

Theorem 3.9 Let $\phi$ be a concave function on $\Re$. Then under the assumptions (A1) and (A2),

(i) if $\phi$ is supermodular, then, for any $\mathbf{a}, \mathbf{b} \in \Re^{n}$,

$$
\mathbf{a} \succeq_{\mathrm{m}} \mathbf{b} \Longrightarrow \sum_{i=1}^{n} \phi\left(X_{n-i+1}, a_{(i)}\right) \geq_{\mathrm{st}} \sum_{i=1}^{n} \phi\left(X_{n-i+1}, b_{i}\right)
$$

(ii) if $\phi$ is submodular, then, for any $\mathbf{a}, \mathbf{b} \in \Re^{n}$,

$$
\mathbf{a} \succeq_{\mathrm{m}} \mathbf{b} \Longrightarrow \sum_{i=1}^{n} \phi\left(X_{i}, a_{(i)}\right) \geq_{\mathrm{st}} \sum_{i=1}^{n} \phi\left(X_{i}, b_{i}\right) .
$$

Corollary 3.10 Let $\phi$ be a concave function on $\Re$. Under the assumptions (A1) and (A2), for any $\mathbf{a}, \mathbf{b} \in \Re^{n}$,

$$
\mathbf{a} \succeq_{\mathrm{m}} \mathbf{b} \Longrightarrow \sum_{i=1}^{n} \phi\left(X_{i}-a_{(i)}\right) \geq_{\text {st }} \sum_{i=1}^{n} \phi\left(X_{i}-b_{i}\right) .
$$

Finally, we give an example where the assumptions (A1) and (A2) are satisfied.

Example 3.11 Let $\boldsymbol{Y}=\left(Y_{1}, \ldots, Y_{n}\right)$ be an $n$-dimensional normal variable with mean vector $\boldsymbol{\mu}=\left(\mu_{1}, \ldots, \mu_{n}\right)$ satisfying $\mu_{1} \leq \mu_{2} \leq \ldots \leq \mu_{n}$, and

$$
\operatorname{Cov}\left(Y_{i}, Y_{j}\right)=\left[\rho+(1-\rho) \delta_{i j}\right] \sigma^{2}, \quad \forall i, j
$$

where $\rho>-\frac{1}{n-1}$ and $\delta_{i j}$ is Kronecker delta, that is $\delta_{i i}=1$ and $\delta_{i j}=0, \forall i \neq j$. Hollander et al. (1977) proved that the joint density function of $\boldsymbol{Y}$ is AI. It is easy to see that the joint density function of $\boldsymbol{Y}$ is log-concave. Thus, the assumptions (A1) and (A2) hold.

\subsection{Increasing convex ordering}

So far, we have obtained all the results under the assumption (A1) that the joint density function is log-concave. However, there exist cases where the joint density functions are not log-concave, even if the marginal density functions are log-concave (cf. You and Li, 2014). An (1998) remarked that if $X$ has a log-concave density, then its density has at most an exponential tail, i.e.,

$$
f(x)=O(\exp (-\lambda x)), \quad \lambda>0, \quad x \rightarrow \infty .
$$


Thus, all the power moments $\mathrm{E}|X|^{\gamma}, \gamma>0$, of the random variable $X$ exist. In this section, we prove the following theorem without the assumption (A1).

Theorem 3.12 Under the assumptions (A2) and (A3),

(i) if $\phi$ is supermodular, then, for any $\mathbf{a}, \mathbf{b} \in \Re^{n}$,

$$
\mathbf{a} \succeq_{\mathrm{m}} \mathbf{b} \Longrightarrow \sum_{i=1}^{n} \phi\left(X_{i}, a_{(i)}\right) \geq_{\mathrm{icx}} \sum_{i=1}^{n} \phi\left(X_{i}, b_{(i)}\right) .
$$

(ii) if $\phi$ is submodular, then, for any $\mathbf{a}, \mathbf{b} \in \Re^{n}$,

$$
\mathbf{a} \succeq_{\mathrm{m}} \mathbf{b} \Longrightarrow \sum_{i=1}^{n} \phi\left(X_{n-i+1}, a_{(i)}\right) \geq_{\mathrm{icx}} \sum_{i=1}^{n} \phi\left(X_{n-i+1}, b_{(i)}\right)
$$

Proof. We only prove part (i) as the proof of part (ii) follows on the same lines. By the nature of majorization, we only need to prove that for all

$$
\left(a_{(1)}, a_{(2)}, c_{3}, \ldots, c_{n}\right) \succeq_{\mathrm{m}}\left(b_{(1)}, b_{(2)}, c_{3}, \ldots, c_{n}\right),
$$

with $\left(a_{(1)}, a_{(2)}\right) \succeq_{\mathrm{m}}\left(b_{(1)}, b_{(2)}\right)$, we have

$$
\phi\left(X_{1}, a_{(1)}\right)+\phi\left(X_{2}, a_{(2)}\right)+\sum_{i=3}^{n} \phi\left(X_{i}, c_{i}\right) \geq_{\mathrm{icx}} \phi\left(X_{1}, b_{(1)}\right)+\phi\left(X_{2}, b_{(2)}\right)+\sum_{i=3}^{n} \phi\left(X_{i}, c_{i}\right) .
$$

We denote

$$
\varphi(\mathbf{x}, \mathbf{a})=\phi\left(x_{1}, a_{1}\right)+\phi\left(x_{2}, a_{2}\right)+\sum_{i=3}^{n} \phi\left(x_{i}, c_{i}\right)
$$

$\mathbf{a}_{12}=\left(a_{(1)}, a_{(2)}\right)$ and $\mathbf{a}_{21}=\left(a_{(2)}, a_{(1)}\right)$. It is sufficient to prove that for all increasing convex function $h$,

$$
\mathrm{E}\left[h\left(\varphi\left(\mathbf{X}, \mathbf{a}_{12}\right)\right)\right] \geq \mathrm{E}\left[h\left(\varphi\left(\mathbf{X}, \mathbf{b}_{12}\right)\right)\right] \text { holds. }
$$

Now,

$$
\begin{aligned}
\mathrm{E} & {\left[h\left(\varphi\left(\mathbf{X}, \mathbf{a}_{12}\right)\right)\right]-\mathrm{E}\left[h\left(\varphi\left(\mathbf{X}, \mathbf{b}_{12}\right)\right)\right] } \\
= & \left(\int \cdots \int_{x_{1} \leq x_{2}}+\int \ldots \int_{x_{1} \geq x_{2}}\right) h\left(\varphi\left(\mathbf{x}, \mathbf{a}_{12}\right)\right) f(\mathbf{x}) d x_{1} \ldots d x_{n} \\
& -\left(\int \cdots \int_{x_{1} \leq x_{2}}+\int \cdots \int_{x_{1} \geq x_{2}}\right) h\left(\varphi\left(\mathbf{x}, \mathbf{b}_{12}\right)\right) f(\mathbf{x}) d x_{1} \ldots d x_{n} \\
= & \int \ldots \int_{x_{1} \leq x_{2}}\left[h\left(\varphi\left(\mathbf{x}, \mathbf{a}_{12}\right)\right)-h\left(\varphi\left(\mathbf{x}, \mathbf{b}_{12}\right)\right)\right] f(\mathbf{x}) d x_{1} \ldots d x_{n} \\
& +\int \ldots \int_{x_{1} \leq x_{2}}\left[h\left(\varphi\left(\mathbf{x}, \mathbf{a}_{21}\right)\right)-h\left(\varphi\left(\mathbf{x}, \mathbf{b}_{21}\right)\right)\right] f\left(\boldsymbol{\pi}_{12}(\mathbf{x})\right) d x_{1} \ldots d x_{n} \\
\geq & \int \ldots \int_{x_{1} \leq x_{2}}\left[h\left(\varphi\left(\mathbf{x}, \mathbf{a}_{12}\right)\right)+h\left(\varphi\left(\mathbf{x}, \mathbf{a}_{21}\right)\right)-h\left(\varphi\left(\mathbf{x}, \mathbf{b}_{12}\right)\right)-h\left(\varphi\left(\mathbf{x}, \mathbf{b}_{21}\right)\right)\right] f\left(\boldsymbol{\pi}_{12}(\mathbf{x})\right) d x_{1} \ldots d x_{n} .
\end{aligned}
$$


The last inequality follows from the fact that $f(\mathbf{x})$ is AI, i.e.,

$$
f(\mathbf{x})-f\left(\boldsymbol{\pi}_{12}(\mathbf{x})\right) \geq 0, \quad \forall x_{1} \leq x_{2} .
$$

Thus, it is sufficient to prove

$$
h\left(\varphi\left(\mathbf{x}, \mathbf{a}_{12}\right)\right)+h\left(\varphi\left(\mathbf{x}, \mathbf{a}_{21}\right)\right)-h\left(\varphi\left(\mathbf{x}, \mathbf{b}_{12}\right)\right)-h\left(\varphi\left(\mathbf{x}, \mathbf{b}_{21}\right)\right) \geq 0
$$

Since $\phi$ is convex and supermodular, for $a_{(1)} \leq b_{(1)} \leq b_{(2)} \leq a_{(2)}$ and $x_{1} \leq x_{2}$, we have

$$
\begin{aligned}
\varphi\left(\mathbf{x}, \mathbf{a}_{12}\right)-\varphi\left(\mathbf{x}, \mathbf{b}_{12}\right)=\phi\left(x_{1}, a_{(1)}\right)+\phi\left(x_{2}, a_{(2)}\right)-\phi\left(x_{1}, b_{(1)}\right)-\phi\left(x_{2}, b_{(2)}\right) \\
=\quad\left[\phi\left(x_{1}, a_{(1)}\right)+\phi\left(x_{1}, a_{(2)}\right)-\phi\left(x_{1}, b_{(1)}\right)-\phi\left(x_{1}, b_{(2)}\right)\right] \\
+\left[\phi\left(x_{2}, a_{(2)}\right)-\phi\left(x_{1}, a_{(2)}\right)+\phi\left(x_{1}, b_{(2)}\right)-\phi\left(x_{2}, b_{(2)}\right)\right] \geq 0 \\
\\
\varphi\left(\mathbf{x}, \mathbf{a}_{12}\right)-\varphi\left(\mathbf{x}, \mathbf{b}_{21}\right)=\phi\left(x_{1}, a_{(1)}\right)+\phi\left(x_{2}, a_{(2)}\right)-\phi\left(x_{2}, b_{(1)}\right)-\phi\left(x_{1}, b_{(2)}\right) \\
=\quad\left[\phi\left(x_{1}, a_{(1)}\right)+\phi\left(x_{1}, a_{(2)}\right)-\phi\left(x_{1}, b_{(1)}\right)-\phi\left(x_{1}, b_{(2)}\right)\right] \\
\quad+\left[\phi\left(x_{2}, a_{(2)}\right)-\phi\left(x_{1}, a_{(2)}\right)+\phi\left(x_{1}, b_{(1)}\right)-\phi\left(x_{2}, b_{(1)}\right)\right] \geq 0
\end{aligned}
$$

and

$$
\begin{aligned}
& \varphi\left(\mathbf{x}, \mathbf{a}_{12}\right)+\varphi\left(\mathbf{x}, \mathbf{a}_{21}\right)-\varphi\left(\mathbf{x}, \mathbf{b}_{12}\right)-\varphi\left(\mathbf{x}, \mathbf{b}_{21}\right) \\
&=\quad \phi\left(x_{1}, a_{(1)}\right)+\phi\left(x_{1}, a_{(2)}\right)-\phi\left(x_{1}, b_{(1)}\right)-\phi\left(x_{1}, b_{(2)}\right) \\
&+\phi\left(x_{2}, a_{(2)}\right)+\phi\left(x_{2}, a_{(1)}\right)-\phi\left(x_{2}, b_{(2)}\right)-\phi\left(x_{2}, b_{(1)}\right) \geq 0 .
\end{aligned}
$$

Thus, for any increasing convex function $h$, if $\varphi\left(\mathbf{x}, \mathbf{a}_{21}\right) \geq \varphi\left(\mathbf{x}, \mathbf{b}_{21}\right)$, then $h\left(\varphi\left(\mathbf{x}, \mathbf{a}_{21}\right)\right) \geq$ $h\left(\varphi\left(\mathbf{x}, \mathbf{b}_{21}\right)\right)$ and $h\left(\varphi\left(\mathbf{x}, \mathbf{a}_{12}\right)\right) \geq h\left(\varphi\left(\mathbf{x}, \mathbf{b}_{12}\right)\right)$, which implies (3.6). Otherwise, if $\varphi\left(\mathbf{x}, \mathbf{a}_{21}\right) \leq$ $\varphi\left(\mathbf{x}, \mathbf{b}_{21}\right)$, we have

$$
\left(\varphi\left(\mathbf{x}, \mathbf{a}_{21}\right), \varphi\left(\mathbf{x}, \mathbf{a}_{12}\right)\right) \succeq_{\mathrm{m}}\left(\varphi\left(\mathbf{x}, \mathbf{b}_{21}\right), \varphi\left(\mathbf{x}, \mathbf{a}_{21}\right)-\left(\varphi\left(\mathbf{x}, \mathbf{b}_{21}\right)-\varphi\left(\mathbf{x}, \mathbf{a}_{12}\right)\right)\right) .
$$

Therefore,

$$
\begin{aligned}
& h\left(\varphi\left(\mathbf{x}, \mathbf{a}_{12}\right)\right)+h\left(\varphi\left(\mathbf{x}, \mathbf{a}_{21}\right)\right) \\
& \quad \geq h\left(\varphi\left(\mathbf{x}, \mathbf{b}_{21}\right)\right)+h\left(\varphi\left(\mathbf{x}, \mathbf{a}_{21}\right)-\left(\varphi\left(\mathbf{x}, \mathbf{b}_{21}\right)-\varphi\left(\mathbf{x}, \mathbf{a}_{12}\right)\right)\right) \\
& \quad \geq h\left(\varphi\left(\mathbf{x}, \mathbf{b}_{21}\right)\right)+h\left(\varphi\left(\mathbf{x}, \mathbf{b}_{12}\right)\right),
\end{aligned}
$$

where the first inequality is due to the convexity of $h$. Therefore, (3.6) holds and the desired result follows. 


\section{An application to optimal capital allocation}

In this section, we outline an application of our main results. Let $X_{1}, \ldots, X_{n}$ be $n$ risks in a portfolio. Assume that a company wishes to allocate the total capital $p=p_{1}+\ldots+p_{n}$ to the corresponding risks. As defined in $\mathrm{Xu}$ and $\mathrm{Hu}$ (2012), the loss function

$$
L(\boldsymbol{p})=\sum_{i=1}^{n} \phi\left(X_{i}-p_{i}\right), \quad \boldsymbol{p} \in A=\left\{\boldsymbol{p} \in \Re_{+}^{n}: p_{1}+\ldots+p_{n}=p\right\}
$$

is a reasonable criterion to set the capital amount $p_{i}$ to $X_{i}$, where $\phi$ is convex. A good capital allocation strategy is to make the loss function $L(\boldsymbol{p})$ as small as possible in some sense. Besides, the different capital allocation strategies affect the general loss function via stochastic comparisons. Therefore, it is meaningful for us to find the best capital allocation strategy if it exists via the methods in Section 3.

Theorem 4.1 If the joint density function of $X_{1}, \ldots, X_{n}$ satisfies assumptions (A1) and (A2) of Section 3, and if $\boldsymbol{p}^{*}=\left(p_{1}^{*}, \ldots, p_{n}^{*}\right)$ is the solution to the best capital allocation strategy, then, we have $p_{1}^{*} \leq p_{2}^{*} \leq \ldots \leq p_{n}^{*}$.

Proof. Let $\boldsymbol{p}=\left(p_{1}, p_{2}, p_{3}, \ldots, p_{n}\right)$ be any admissible allocation, and let $\hat{\boldsymbol{p}}=\left(p_{2}, p_{1}, p_{3}, \ldots, p_{n}\right)$. Without loss of generality, we assume $p_{1} \leq p_{2}$. By the nature of majorization, we only need to prove that

$$
P(L(\hat{\boldsymbol{p}}) \geq t) \geq P(L(\boldsymbol{p}) \geq t), \quad \forall t .
$$

That means

$$
\phi\left(X_{1}-p_{2}\right)+\phi\left(X_{2}-p_{1}\right)+\sum_{i=3}^{n} \phi\left(X_{i}-p_{i}\right) \geq_{\text {st }} \phi\left(X_{1}-p_{1}\right)+\phi\left(X_{2}-p_{2}\right)+\sum_{i=3}^{n} \phi\left(X_{i}-p_{i}\right) .
$$

Since the usual stochastic order is closed under convolution, we only need to prove

$$
\phi\left(X_{1}-p_{2}\right)+\phi\left(X_{2}-p_{1}\right) \geq_{\text {st }} \phi\left(X_{1}-p_{1}\right)+\phi\left(X_{2}-p_{2}\right) .
$$

Under assumptions (A1) and (A2), (4.1) holds due to Corollary 3.8. Therefore, the desired conclusion follows.

\section{Acknowledgements}

The authors would like to sincerely thank the anonymous referee for the constructive comments and suggestions which led to an improvement of the paper. Besides, we are grateful to Professor Taizhong $\mathrm{Hu}$ and Professor Maochao Xu for their comments and contributions to this paper.

The first author is supported by the NNSF of China (No. 11401558), the Fundamental Research Funds for the Central Universities (No. WK2040160010) and China Postdoctoral 
Science Foundation (No. 2014M561823). The second author is Supported by the NNSF of China (Nos. 11201452 and 11271346).

\section{References}

[1] An, M.Y. (1998). Logconcavity versus logconvexity: a complete characterization. Journal of Economic Theory, 80, 350-369.

[2] Cai, J and Wei, W (2014). Some new notions of dependence with applications in optimal allocation problems. Insurance: Mathematics and Economics, 55, 200-209.

[3] Eaton, M.L. (1982). A review of selected topics in multivariate probability inequalities. Annals of Statistics, 10, 11-43.

[4] Hollander, M., Proschan, F. and Sethuraman, J. (1977). Functions decreasing in transposition and their applications in ranking problems. The Annals of Statistics, 5, 722-733.

[5] Karlin, S. and Rinott, Y. (1983). Comparison of measures, multivariate majorization, and applications to statistics. In: S. Karlin et al. (Eds.), Studies in Econometrics, Time Series, and Multivariate Statistics, Academic Press, New York, pp.465-489.

[6] Ma, C. (2000). Convex orders for linear combinations of random variables. Journal of Statistical Planning and Inference, 84, 11-25.

[7] Mao, T., Pan, X. and Hu, T. (2013). On orderings between weighted sums of random variables. Probability in the Engineering and Informational Science, 27, 85-97.

[8] Marshall, A.W., Olkin, I. and Arnold, B.C. (2011). Inequalities: Theory of Majorization and Its Applications. Springer, New York.

[9] Müller, A. and Stoyan, D. (2002). Comparison Methods for Stochastic Models and Risks. John Wiley \& Sons, Ltd., West Sussex.

[10] Pan, X., Xu, M. and Hu, T. (2013). Some inequalities of linear combinations of independent random variables: II. Bernoulli, 19(5A), 1776-1789.

[11] Prékopa, A. (1973). On logarithmic concave measures and functions. Acta Sci. Math. (Szeged), 34, 335-343.

[12] Shanthikumar, J.G. and Yao, D.D. (1991). Bivariate characterization of some stochastic order relations. Advances in Applied Probability 23, 642-659. 
[13] Xu, M. and Hu, T. (2011). Some inequalities of linear combinations of independent random variables: I. Journal of Applied Probability, 48, 1179-1188.

[14] Xu, M. and $\mathrm{Hu}, \mathrm{T}$. (2012). Stochastic comparisons of capital allocations with applications. Insurance: Mathematics and Economics, 50, 293-298.

[15] Shaked, M., and Shanthikumar, J.G. (2007). Stochastic Orders. Springer, New York.

[16] You, Y. and Li, X. (2014). Optimal capital allocations to interdependent actuarial risks. Insurance: Mathematics and Economics, 57, 104-113.

[17] Yu, Y. (2011). Some stochastic inequalities for weighted sums. Bernoulli, 17, 1044-1053. 